\title{
Quench Characteristics of HTS Conductors at Low Temperatures
}

\author{
Taotao Huang, Elena Martínez, Chris Friend, and Yifeng Yang
}

\begin{abstract}
Not withstanding their high stability at liquid nitrogen temperatures, quench propagation due local disturbances remains a crucial issue for high temperature superconductors (HTS) operating at low temperatures as high field insert coils and cryogen-free magnets. In contrast to low temperature superconductors (LTS), normal zone in HTS at low temperatures must develop over a much extended temperature range for current sharing with a highly non-linear dependence on temperature. The present work addresses this issue directly using both computer simulation and experimental studies. Several quench characteristics unique to HTS have identified using a comparative study of the underlying quasilinear parabolic equation represented in an appropriate dimensionless form for proper isolation of the known effects of temperature dependence of thermal properties. Most notable and counter-intuitive finding is that the minimum propagation zone (MPZ) was found to increase with transport current. The correlations of minimum quench energy (MQE) and the quench temperature of MPZ with operating temperature and current have been obtained for comparison with experimental results.
\end{abstract}

Index Terms-High temperature superconductors, quench protection, superconducting magnets.

\section{INTRODUCTION}

$\mathbf{H}$ IGH temperature superconductor insert coil is the only option available for building all-superconducting magnets above $25 \mathrm{~T}$. While material performance and mechanical design remain serious challenges for HTS insert coils, cryogenic stability and quench management have also coming into sharp focus following recent progress in 2nd generation YBCO tapes and Bi2212 wires.

HTS high field insert magnets are design to operate at liquid helium temperatures for achieving maximum field and current performance. Unlike their counterparts at liquid nitrogen temperatures, HTS coils at helium temperatures do not enjoy the extended thermal stability due to a much reduced heat capacity and a higher operating current density. Compared to low temperature superconductors (LTS), the quench behavior of HTS

Manuscript received August 28, 2007. This work was supported in part by Oxford Instruments Nanoscience and European Union FP6 NMP3-CT2004505724.

T. Huang and Y. Yang are with the Institute of Cryogenics, School of Engineering Sciences, University of Southampton, Southampton SO17 1BJ, U.K. (e-mail: y.yang@soton.ac.uk).

E. Martínez is with Instituto de Ciencia de Materiales de Aragón, CSIC-Universidad de Zaragoza, C/María de Luna 3, 50018 Zaragoza, Spain (e-mail: elenamar@unizar.es).

C. Friend is with Oxford Instruments NanoScience, Tubney Woods, Abingdon, Oxon OX13 5QX, U.K. (e-mail: chris.friend@oxinst.com).

Digital Object Identifier 10.1109/TASC.2008.920797 at low temperatures is far more complex for the following reasons [1]-[3]. First of all, the current-share regime, fundamental to the development of the normal zone, is extended from just a few Kelvin for LTS to up to $100 \mathrm{~K}$ for HTS at helium temperatures. Within this extended current-share regime, the E-J characteristics of the HTS are highly nonlinear with temperature and cannot be approximated by the classical critical state model. Hence the heat generation in the current share regime is a complex nonlinear function of temperature, instead of the linear dependence found in LTS. Secondly, the relevant thermal-electrical properties including resistivity, specific heat and thermal conductivity also exhibit a strong dependence on temperature in the extended current share regime. Such coupled nonlinearities have the potential to fundamentally change the behavior of the underlying heat diffusion process during the quench onset and subsequent propagation.

\section{Dimensionless HeAT Diffusion EQUATION FOR QUENCH ANALYSIS}

Without loosing fundamental generality while maintaining simplicity, the present work is confined to one dimensional stabilized superconducting composite in adiabatic conditions. The heat diffusion equation relevant for the quench process is simply:

$$
\rho c_{p}(T) \frac{\partial}{\partial t} T(x, t)=\frac{\partial}{\partial x}\left(k(T) \frac{\partial}{\partial x} T(x, t)\right)+G(J, T)
$$

where $\rho, c_{\mathrm{p}}, k$ are density, specific heat and thermal conductivity respectively. The heat generation $G(J, T)$ is due to the current share in the normal matrix. By convention, the transport current density $J$ is measured against the superconducting cross-section, which has a fill factor of $\lambda$. The heat generation during the quench process is determined by the electric field $E$ developed along the superconductor as the result of current sharing between the superconductor and the stabilization matrix, i.e, $G(J, T)=\lambda J E(J, T)$. As the dissipation in LTS can adequately be described by the classic critical state model, the current is shared by the superconductor carrying the critical current $J_{\mathrm{c}}(T)$ and the normal matrix the remainder current $J-J_{\mathrm{c}}(T)$ at a voltage of $\lambda(1-\lambda)^{-1} \rho_{\mathrm{m}}(T)\left(J-J_{\mathrm{c}}(T)\right)$. With a linear critical current $J_{\mathrm{c}}(T)=J_{\mathrm{c} 0}\left(1-T / T_{\mathrm{c}}\right)$, the heat generation is also a linear function of $T$ above the current-share temperature $T_{\mathrm{cs}}=T_{\mathrm{c}}\left(1-J / J_{\mathrm{c} 0}\right)$ :

$$
G_{C R}= \begin{cases}\lambda^{2}(1-\lambda)^{-1} \rho_{m} J J_{c 0}\left(T-T_{c s}\right) T_{c}^{-1}, & T \geq T_{c s} \\ 0, & T<T_{c s}\end{cases}
$$


For HTS materials, the classic critical state model has to be replaced by a more general power-law E-J characteristic: $E(J, T)=E_{0}\left(J / J_{\mathrm{c}}(T)\right)^{n(T)}$. Both the critical current $J_{\mathrm{c}}$ and the power exponent $n$ are typically nonlinear functions of temperature, and the electric field $E(J, T)$ of current-share between the HTS and the normal matrix has to be determined by a nonlinear algebraic equation [5]:

$$
J=J_{c}(T)\left(\frac{E(J, T)}{E_{0}}\right)^{\frac{1}{n(T)}}+\frac{1-\lambda}{\lambda \rho_{m}(T)} E(J, T) .
$$

The heat generation $G(J, T)=\lambda J E(J, T)$ is obtained by solution of (3).

For HTS operating at a given temperature $T_{0}$, the following dimensionless terms can be defined:

$$
\theta=\frac{T-T_{0}}{T_{c}-T_{0}}, \quad \xi=x / L, \quad \tau=t / L^{2}\left(\rho c_{p}\left(T_{0}\right) / k\left(T_{0}\right)\right)
$$

where $L$ is the half length of the conductor. Substitution of (4) into (1) results in a non-dimensional diffusion equation:

$\gamma(\theta) \frac{\partial}{\partial \tau} \theta(\xi, \tau)=\frac{\partial}{\partial \xi}\left(\kappa(\theta) \frac{\partial}{\partial \xi} \theta(\xi, \tau)\right)+\frac{G(J, \theta)}{k\left(T_{0}\right)\left(T_{c}-T_{0}\right) L^{-2}}$

where $\gamma=c_{\mathrm{p}}(T) / c_{\mathrm{p}}\left(T_{0}\right)$ and $\kappa=k(T) / k\left(T_{0}\right)$. The boundary conditions of the dimensionless (5) are $\theta(1, \tau)=0$ and $\theta_{\xi}(0, \tau)=0$.

While (5) has a similar appearance as the dimensioned (1), it offers much easier comparison between HTS and LTS. For example, when compare HTS at nitrogen temperature and LTS at helium temperature, it becomes obvious that the main difference is only in the time constant $L^{2} \rho c_{p} / k$. The much increase heat capacity in HTS only slows down the quench process and increases the quench energy, while the quench behavior of HTS at high temperature remains fundamentally the same as LTS.

The term $G_{0}=k\left(T_{0}\right)\left(T_{\mathrm{c}}-T_{0}\right) / L^{2}$ represents the heat conduction from the normal zone to the end of the conductor at the operating temperature $T_{0}$. For superconductors obeying the critical state model, an extended temperature range for currentshare does not affect the linear behavior of generation $G(J, \theta)$ given by (2). For HTS at low temperatures, a normalized $G(J, \theta)$ by $G_{0}$ to a large extent eliminates the heat conduction effect from the non-linear current-share heat generation over a large temperature range described by (3).

\section{METHODS}

In the present work (5) was solved numerically in order to investigate the HTS quench at low temperatures, with focus on the nonlinear current-share due to power-law E-J characteristics and the temperature dependence of thermal properties over a extended temperature range for current sharing. Point disturbance of finite energy was implemented as initial conditions of a localized hot zone less than the minimum propagation zone (MPZ).

The verification of the numerical results on the non-linear current share for HTS materials was beyond the scope of the present work due the very long sample length required. It was found,

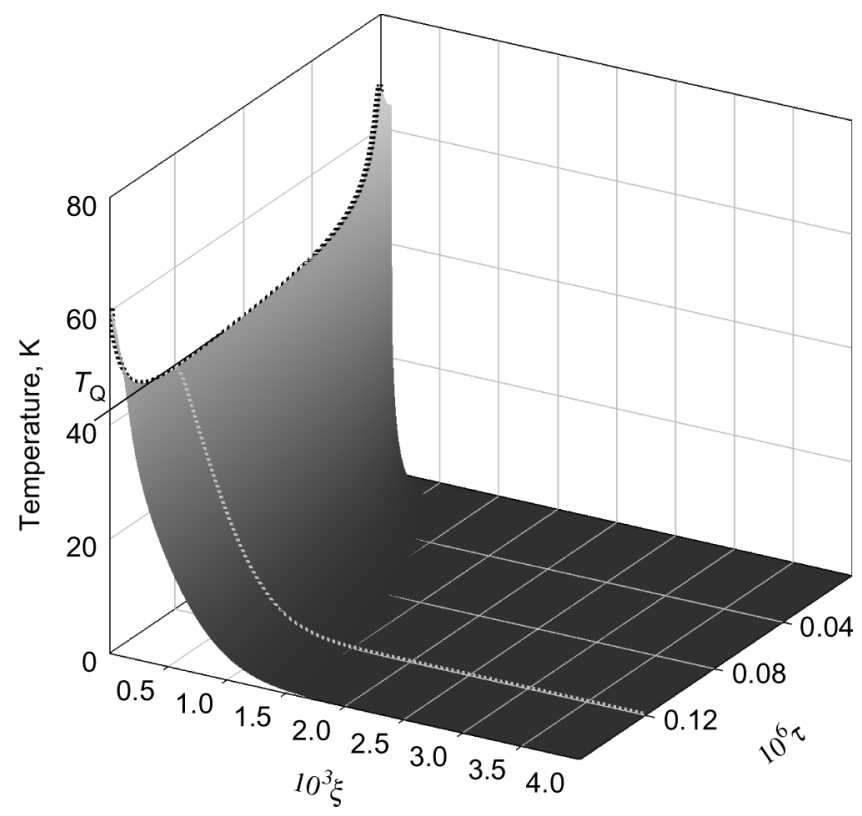

Fig. 1. Spatial-temporal profile of a quench process following a point disturbance.

however, that a similar behavior could be seen in $\mathrm{MgB}_{2}$ conductors at a moderate sample length. Confirmation of the numerical results was obtained therefore by direct quench measurements [6] carried out on a commercial stabilized $\mathrm{MgB}_{2}$ tape.

\section{RESULTS AND DISCUSSION}

The quench process following a point disturbance has a typical spatial-temporal distribution shown in Fig. 1. When the deposited energy is significantly close to the minimum quench energy (MQE), a quasi-steady-state may be sustained for a significant period (black dotted line), and the corresponding spatial temperature distribution (grey dotted line) can be considered a close approximation of the minimum propagation zone MPZ. It should be noted that the MPZ is only about $0.2 \%$ of the conductor length $L$, which should be set significantly longer to minimize the finite length effect. Only $0.4 \%$ of the sample length is shown in Fig. 1.

The maximum temperature of the MPZ is known as the quench temperature $T_{\mathrm{Q}}$. For HTS at low temperatures, $T_{\mathrm{Q}}$ is expected somewhere in the middle of the extension temperature range for current-share and can be significantly lower than the critical temperature $T_{\mathrm{c}}$. Although an explicit expression of $T_{\mathrm{Q}}$ is undetermined at present, the quench temperature decreases towards $T_{\mathrm{cs}}$ with increasing either the transport current or the conductor length.

\section{A. Heat Generation in Nonlinear Current Share and Minimum Propagation Zone (MPZ)}

In Fig. 3, the heat generation of the nonlinear current share in HTS is compared with the linear current share of the critical state. In the upper pane, the heat generation is plotted as a function of both current and temperature. The linear plane in corresponds to the critical state with constant resistivity, while the curved surface below is for the power-law E-J characteristics. The lower pane of Fig. 2 shows in detail the current share 

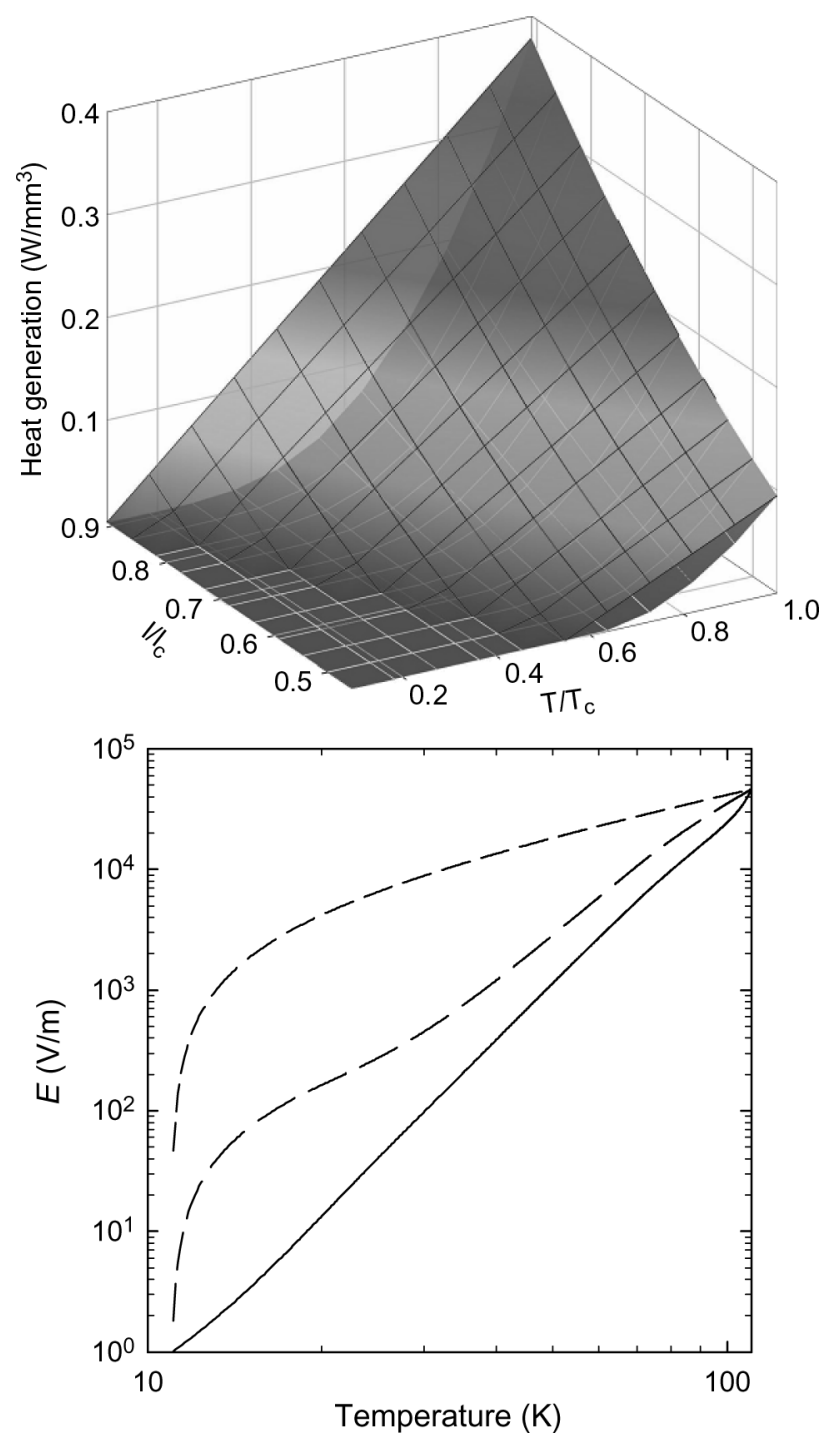

Fig. 2. Upper pane: heat generation as a function of temperature and current for the critical state (top plane) and power-law $E(J)$ (bottom surface); lower pane: current share voltage for HTS at $4.2 \mathrm{~K}$ and $J=0.9 J_{\mathrm{c} 0}$ with a power-law E-J and $\rho_{\mathrm{m}}(T)$ (solid line), critical state and constant $\rho_{\mathrm{m}}$ (dashed line), and critical state with $\rho_{\mathrm{m}}(T)$ (long dashed line).

voltage of HTS at $4.2 \mathrm{~K}$ and $J=0.9 J_{\mathrm{c} 0}$. The solid line is for a power-law $E(J)$ with a temperature dependent $\rho_{\mathrm{m}}$ of RRR $=$ 100 , the dashed line at the top for the critical state of $n=\infty$ and constant resistive of $\rho_{\mathrm{m}}\left(T_{\mathrm{c}}\right)$, and the long dashed line in the middle for the critical state with $\rho_{\mathrm{m}}(T)$.

The log-log plot illustrates clearly that when quenching at low temperatures, the HTS heat generation is up to two orders of magnitude lower than in the critical state. Such a drastic reduction in the heat generation is certain to have a profound effect on the length of the minimum propagation zone, which is given by [1], [4]:

$$
L_{M P Z}=\pi \sqrt{\frac{k\left(T_{Q}-T_{C S}\right)}{G\left(J, T_{Q}\right)}} .
$$

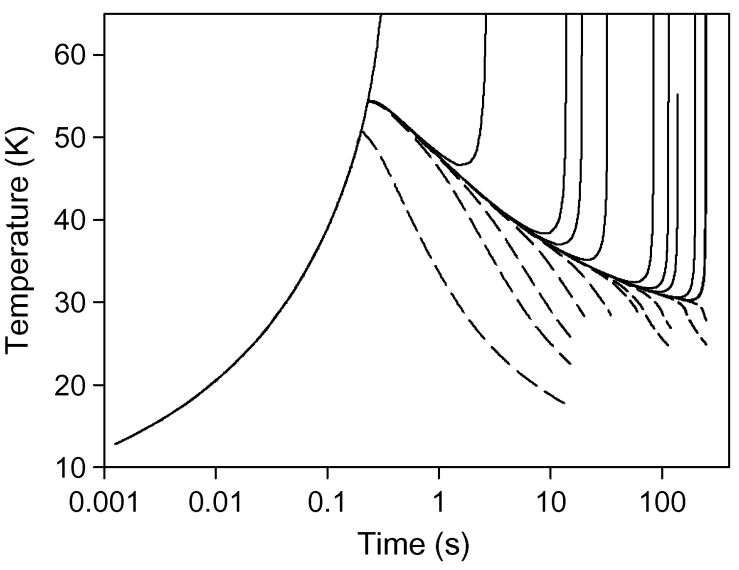

Fig. 3. Quench initiation for HTS at $10 \mathrm{~K}$ and carrying 55\% $J_{\mathrm{co}}$ by different quench energy refined to approach MQE with successive increase of number of significant digits.

With a significant lower $G(J, T)$, HTS must either quench at a rather high quench temperature $T_{\mathrm{Q}}$ or develop a much longer MPZ to quench at a low $T_{\mathrm{Q}}$.

\section{B. Quench Temperature $T_{Q}$ for HTS at Low Temperatures}

Fig. 3 shows the numerical results for the quench onset/recovery in a HTS conductor at $10 \mathrm{~K}$ and carrying a moderate current of $55 \%$ of the critical current density $2.5 \times 10^{8} \mathrm{~A} / \mathrm{m}^{2}$, a realistic situation for HTS high field insert magnets operating in a background field $>20 \mathrm{~T}$. The set of curves correspond to refined minimum quench energy values with increasing number of significant digits up to 14 . The dashed curves correspond to quench energy just below MQE at respective number of significant digits. It is remarkable that the quench temperature can change as much as $10 \mathrm{~K}$ while the quench energy is practically constant and within $0.1 \%$ of MQE.

In contrast, the "true" quench temperature can be reached with the MQE with six significant digits when the transport current is increased from $55 \%(\bullet)$ to $80 \%(\nabla)$ of $J_{\mathrm{c}}$. $T_{\mathrm{Q}}-T_{\mathrm{cs}}$ is lowered from $10 \mathrm{~K}$ at $0.55 J_{\mathrm{c}}$ to $0.4 \mathrm{~K}$ at $0.8 J_{\mathrm{c}}$.

\section{Minimum Propagation Zone at Different Transport Current}

According to (6), the wide range of $T_{\mathrm{Q}}$ for a very small change in quench energy can result in a significant variation in the length of the normal zone. Fig. 5 shows the corresponding numerical results of MPZ length for successive refinement of quench energy toward MQE. Initial reduction of quench energy towards MQE leads to an increase in the MPZ length to a peak value as the heat generation $G$ reduces sharply (Fig. 2). However, further refinement of quench energy results in a reduction of the normal zone length towards an equilibrium value. The reduction is due to a diminishing $T_{\mathrm{Q}}-T_{\mathrm{cs}}$. It is worth noting that the longer MPZ for high current than low current, contrary to the prediction by the classical quench model.

Using the results in Fig. 4 and Fig. 5, it is possible to plot the length of the propagation zone as a function of $T_{\mathrm{Q}}-T_{\mathrm{cs}}$ as shown in Fig. 6. It is remarkable that for different transport current, the correlation between $L_{\mathrm{MPZ}}$ and $T_{\mathrm{Q}}-T_{\mathrm{cS}}$ remains very similar. With reference to (5), it suggests that the heat generation 


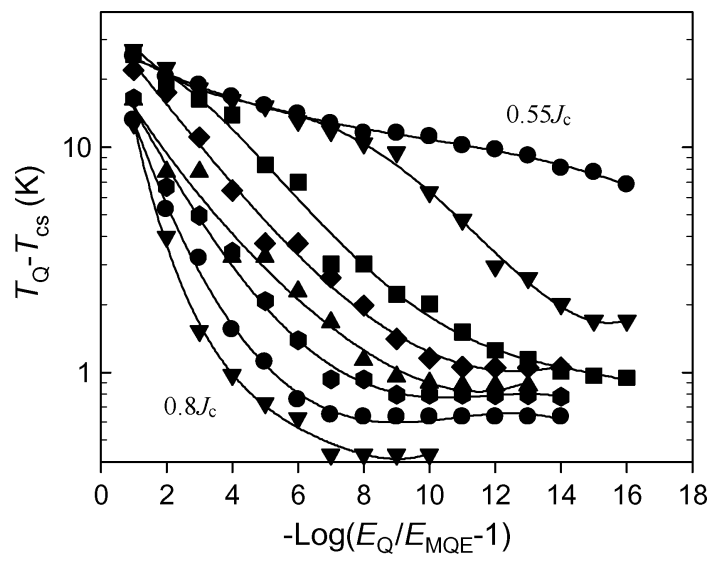

Fig. 4. Quench temperature $T_{\mathrm{Q}}$ above the current share temperature $T_{\mathrm{cs}}$ for different transport current from $55 \%$ to $80 \%$ of $J_{\mathrm{c}}$ at $3.3 \%$ increments. Higher $T_{\mathrm{Q}}-T_{\mathrm{cs}}$ corresponds to lower transport current.

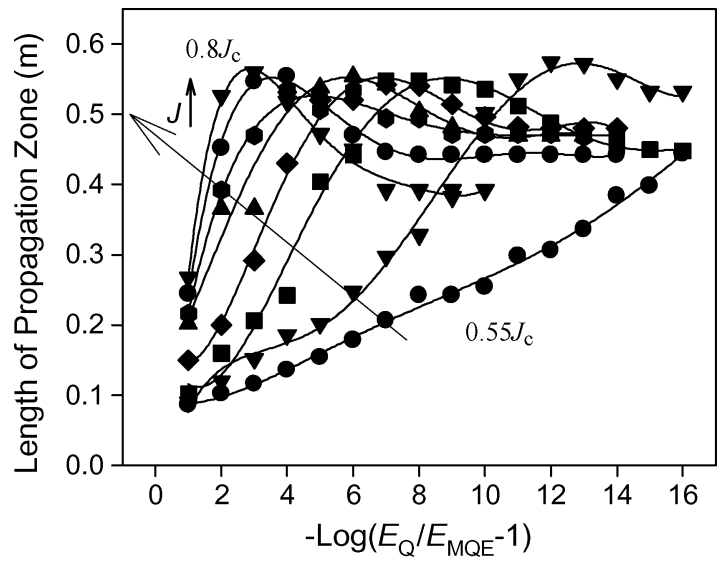

Fig. 5. Variation of MPZ length for different transport current as the quench energy is refined toward MQE.

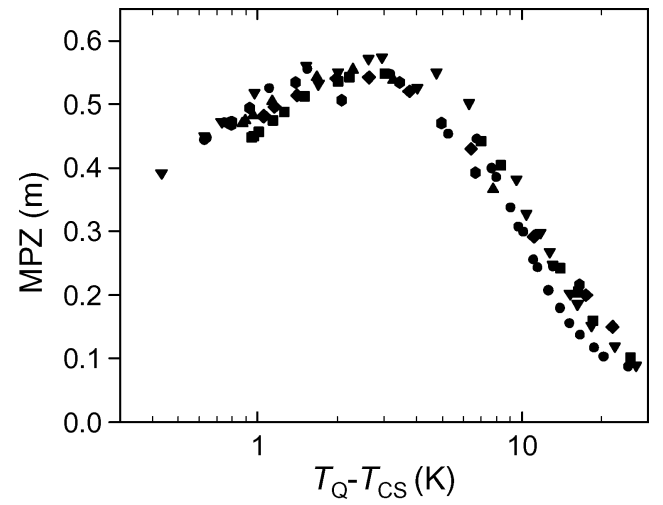

Fig. 6. Correlation between MPZ length and $T_{\mathrm{Q}}-T_{\mathrm{cs}}$ almost independent of transport current.

mainly determined by $T_{\mathrm{Q}}-T_{\mathrm{cS}}$ or MPZ length but independent of the transport current.

\section{Experimental Evidence}

One of the important consequences of numerical quench modeling results present above is that at low transport current

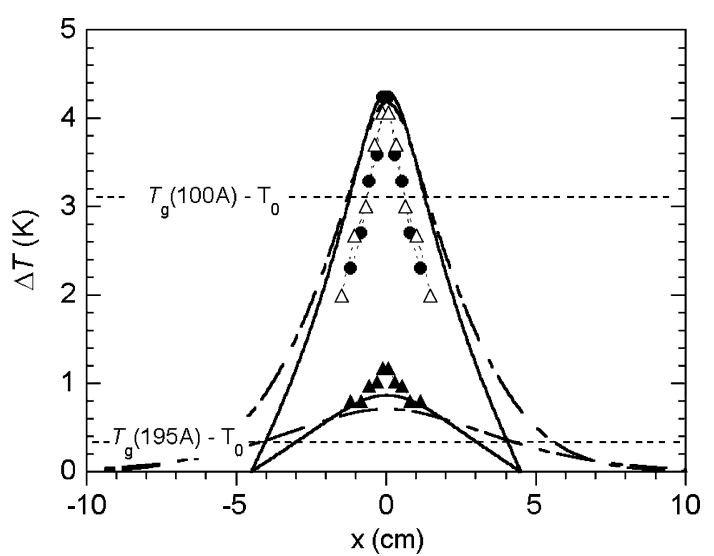

Fig. 7. Measured and calculated MPZ for $\mathrm{MgB}_{2}$ conductor at $27 \mathrm{~K}$ carrying $0.5 I_{\mathrm{C}}$ and $0.95 I_{\mathrm{c}}$ respectively. The solid and dashed lines are the corresponding MPZ profiles obtained numerically for sample lengths of $10 \mathrm{~cm}$ and $20 \mathrm{~cm}$.

the MPZ can be significantly smaller than that at high current, once the quench energy is within $0.1 \%$ of MQE (Fig. 5, compare $55 \% J_{\mathrm{c}}$ and $80 \% J_{\mathrm{c}}$ ). Such behavior is unique to HTS of power-law $E(J)$ as the heat generation reduces sharply at a decreasing quench temperature above $T_{\mathrm{cs}}$ with increasing current. However the length of MPZ up to $0.5 \mathrm{~m}$ long makes experimental verification rather difficult. Instead, we carried out quench measurement on $\mathrm{MgB}_{2}$ conductors, which also follows a power-law $E(J)$. The results shown in Fig. 7 indicate clearly that with $I=195 \mathrm{~A}=0.95 I_{\mathrm{c}}$, all the thermometers are above $T_{\mathrm{cs}}$ within the normal zone, while only two thermometers are in the normal zone for $I=100 \mathrm{~A}=0.5 I_{\mathrm{C}}$.

\section{CONCLUSION}

Numerical quench model for HTS at low temperatures revealed significant difference in the quench behavior compared to LTS. Most significant of these are (1) considerable variation in quench temperature and MPZ length for a well defined MQE and (2) MPZ is longer at high transport current. Power-law E-J over a large current share range is attributed as the underlying mechanism and confirmed by measurement on $\mathrm{MgB}_{2}$.

\section{REFERENCES}

[1] M. N. Wilson, Superconducting Magnets. Oxford: Clarendon, 1983.

[2] Y. Iwasa, Case Studies in Superconducting Magnets. New York: Kluwer Academic, 1994.

[3] L. Dresner, Stability of Superconductors. New York: Kluwer Academic, 2002.

[4] R. B. Banks, Growth and Diffusion Phenomena: Mathematical Frameworks and Applications. Berlin: Springer, 1994.

[5] T. Huang, A. Johnston, Y. Yang, C. Beduz, and C. Friend, "Finite element modeling of thermal stability and quench propagation in a pancake coil of PbBi2223 tapes," IEEE Trans. Appl. Supercond., vol. 15, pp. 1647-1650, Jun. 2005.

[6] E. Martínez, F. Lera, M. Martinez-López, Y. Yang, S. I. Schlachter, P. Lezza, and P. Kováč, "Quench development and propagation in metal/ $\mathrm{MgB}_{2}$ conductors," Supercond. Sci. Technol., vol. 19, pp. 143-150, Jan. 2006. 